\title{
Mirabegron as adjuvant treatment for patients with interstitial cystitis/bladder pain syndrome
}

Michael Di Lena; Victoria Tolls; Kerri-Lynn Kelly; J. Curtis Nickel

Department of Urology, Queen’s University, Kingston, ON, Canada

Cite as: Can Urol Assoc J 2017 Dec. 22; Epub ahead of print. http://dx.doi.org/10.5489/cuaj.4325

Published online December 22, 2017

$* * *$

\begin{abstract}
Introduction: Interstitial cystitis/bladder pain syndrome (IC/BPS) patients represent a heterogeneous group with pain and urinary storage symptoms and varying responses to current treatment options. The novel beta-3 agonist, mirabegron, has been shown to improve storage symptoms of patients with bladder overactivity; however, its effect on symptoms in the IC/BPS population has yet to be studied.

Methods: Patients diagnosed at a single IC centre with IC/BPS undergoing standard therapy were treated with additional daily mirabegron $25 \mathrm{mg}$ and seen in followup post-treatment. Patients completed the Interstitial Cystitis Symptom Index and Problem Index (ICSI/ICPI), and the Pelvic Pain and Urgency/Frequency Patient Symptom Scale (PUF) prior to and following mirabegron treatment. Global (NRS) and symptom-specific outcomes were assessed by comparing the pre- and post-treatment mean scores using tailed-t test $(\mathrm{p}<0.05$ considered statistically significant).
\end{abstract}

Results: A total of 23 patients were available for review pre- and post-mirabegron treatment. There was no significant difference in ICSI ( $p=0.448)$, ICPI ( $\mathrm{p}=0.352)$, or PUF ( $\mathrm{p}=0.869)$ preand post-treatment. Analysis of symptom-specific outcomes show statistically significant improvements in urgency ( $\mathrm{p}=0.048$ ); however, no statistically significant improvements in frequency $(p=0.951)$ or pain $(p=0.952)$ were observed with mirabegron therapy.

Conclusions: IC/BPS patients treated with mirabegron had improvement of urinary urgency, but no significant benefit in terms of pain or urinary frequency. This data suggests that mirabegron's role in the IC/BPS patient should be that of adjuvant treatment to ameliorate urgency. 


\section{Introduction}

Interstitial cystitis (IC), or bladder pain syndrome (BPS), is a chronic disease characterized by suprapubic pain related to bladder filling, coupled with additional symptoms, including increased urinary frequency or urgency, without proven urinary infection or obvious pathology ${ }^{1-3}$. The symptoms typically associated with IC often overlap with other gynecologic and urologic conditions, including overactive bladder (OAB), which is characterized by urinary urgency, with or without urge urinary incontinence, usually with frequency and nocturia but no pain. ${ }^{4}$ Patients diagnosed with IC/BPS comprise a diverse group of patients with varied clinical phenotypes ${ }^{5-7}$ and the presence of various other co-morbid chronic pain and symptom-based syndromes has been widely reported. ${ }^{8-10}$ The heterogeneity of this patient population suggests that IC/BPS, instead of existing as one diagnosable entity is more likely a combination of etiologies with a varied pathogenesis and symptom patterns. Given its diverse clinical phenotype, appropriately managing these patients' symptoms has historically been difficult and the current goals of treatment are largely based on symptomatic relief. At present, a number of treatments are available for IC/BPS which includes conservative measures, oral medical therapy, intravesical drug instillation, hydrodistention, neuromodulation, and surgical therapy. ${ }^{11}$ Although several therapies have been shown to help the symptoms of IC/BPS in clinical trials, multimodal therapy may be the most effective approach in dealing with the range of IC symptoms. ${ }^{12}$

One of the more common and bothersome symptoms in the IC/BPS population is that of urinary urgency ${ }^{13}$. As urgency is considered to be the characteristic symptom of overactive bladder, its presence often confounds and delays the diagnosis and treatment of IC/BPS. Given the reports of qualitative differences in the urgency experienced by IC/BPS patients compared to OAB patients ${ }^{14-15}$, the pathophysiology behind this symptoms development may not be similar. Although, anticholinergics are well established as pharmacotherapy for reducing $\mathrm{OAB}$ symptoms ${ }^{16}$ classic anticholinergic and bladder antispasmodics have shown not to be efficacious in regards to these symptoms in the IC/BPS population ${ }^{17-18}$.

The novel bladder antispasmodics, mirabegron, targets an alternative pathway to achieve control of urinary storage dysfunction. Recent advances in the understanding of the physiopathology of OAB have identified three subtypes of b-adrenoceptor (b1, b2, and b3) in the detrusor muscle and urothelium ${ }^{19-21}$ of which b3-adrenoceptors predominate. The stimulation of the b3 subtype is thought to be the main subtype responsible for mediating relaxation of the detrusor in humans. ${ }^{22}$ Mirabegron is the first b3-adrenoceptor agonist to enter clinical practice, and has been approved for the treatment of symptoms of OAB including urinary frequency, urgency and urge incontinence. As mirabegron has been shown to be successful in treating the urgency and frequency symptoms in patients with $\mathrm{OAB}$, it may also provide a potential efficacious treatment for the IC/BPS patient population. To our knowledge, the use of mirabegron has yet to be reported in this specific population. Our objective was to provide preliminary real life clinical practice data of effectiveness outcomes of mirabegron in patients with IC/BPS. 


\section{Methods}

\section{Participants and study design}

The current report is a prospective evaluation of IC/BPS patients treated at a single outpatient clinic in a real life clinical practice setting. This study was an open-label design where the patients were not blinded to treatment. Clinical features of our patients included chronic (for at least 6 months) pelvic pain, pressure, or discomfort, perceived to be related to the urinary bladder accompanied by at least one other urinary symptom, such as persistent urge to void or frequency. Patients fulfilled the recent IC/BPS definition in the AUA ${ }^{11}$ guidelines.

Exclusion criteria included vesical and urethral pathologies (urinary tract infection, neurogenic bladder, urethral diverticula, bladder or urethral cancer, urinary stones, urge/stress incontinence, pelvic prolapses) or nonurological pathologies (vaginitis, uterine, vaginal or cervical cancers, endometriosis) not related to IC/BPS, or previous/current treatment with mirabegron prior to enrollment.

Patients with IC/BPS with bothersome storage symptoms that did not respond to an anticholinergic trial were offered mirabegron $25 \mathrm{mg}$ daily as add on therapy. Patients continued on standard therapy as prescribed. As part of the clinic protocol, all patients underwent standard assessment which includes history and physical exam and completion of validated questionnaires before and after any treatment intervention. Prospective data was collected as part of our ongoing IRB approved quality assurance audit process. Patients were de-identified before inclusion in this protocol data base.

Patients were re-assessed at the time of their routine clinic appointments and participants underwent standardized clinic repeat assessment and completion of IC/BPS questionnaires.

\section{Measures}

\section{Demographics and self-reported symptom history}

Demographics, self-reported symptom history (including duration of IC/BPS symptoms) and concurrent medications were collected as standard clinic routine.

\section{Condition-specific symptom questionnaires}

IC/BPS specific symptoms were evaluated by the validated ICSI (Interstitial Cystitis Symptom Index), ICPI (Interstitial Cystitis Problem Index) ${ }^{23}$, PUF (Pelvic Pain and Urgency/Frequency Patient Symptom Scale) ${ }^{24}$, NRS pain, NRS urgency, NRS frequency scores. These instruments are routinely collected before and after any treatment change. Pre and post treatment questionnaires were analyzed and reported with a global score. In addition, questions corresponding to specific symptom parameters (pain, urinary urgency, urinary frequency) were identified and scores pre and post treatment were assessed. 


\section{Data analysis}

Statistical analysis was completed using Microsoft Excel 2010 Data Analysis package and R version 3.3.1. All questionnaire scores collected at first post-treatment visit was compared to mean baseline scores using a Wilcoxon ranked-sign test assuming unequal variances. All results were regarded as statistically significant at a p value of 0.05 .

\section{Results}

\section{Patient demographics}

Twenty-eight patients completed standardized clinic questionnaires before and after mirabegron add-on treatment was initiated. Of the twenty-eight patients, five were prescribed but did not take mirabegron and were excluded from final analysis. Patient demographics for the 23 IC/BPS patients are depicted in Table 1. Patients were predominantly female ( $\mathrm{n}=22,95.7 \%)$ and caucasian ( $\mathrm{n}=22,95.7 \%$ ) with a mean age of 49.0 years. Standard duration between pre and post treatment assessments was 6.9 months. Of the patients who had information available regarding length of symptom duration $(\mathrm{n}=15)$, the average IC/BPS patient had experienced symptoms 10.5 months prior to mirabegron treatment.

\section{Concurrent therapy of BPS/IC patients pre- and post-treatment}

Table 2 lists the concomitant treatments of patients for their IC/BPS symptoms during mirabegron treatment period. All patients $(\mathrm{n}=23)$ enrolled in the study were taking first and second line treatment recommendations as outline by AUA guidelines. Of note, one patient stopped intravesical lidocaine and another initiated botox injections during the course of the study.

Mirabegron was well tolerated by the IC/BPS patient population. All patients who initiated mirabegron therapy remained compliant on the medication during the course of the study. Of the twenty-three patients enrolled, 4 patients noted adverse effects believed by patient to be caused by mirabegron treatment. One patient noted increase in blood pressure, another reported headaches, and two noted increased difficulty voiding.

\section{Global and symptom specific questionnaire scores pre- and post-treatment}

Table 3 lists the pre and post treatment total score of the questionnaires. There was no significant difference in total scores of ICSI, ICPI, PUF of patients pre and post treatment with mirabegron. When subcategorizing the PUF questionnaire to symptom score and bother score, no statistically significant difference was identified following treatment.

In terms of discrete symptoms measures (Table 4 ), there was a statistically significant improvement in urgency measures gathered from the ICSI questionnaire $(p=0.0102)$. No statistically significant difference was seen in the pain $(\mathrm{p}=0.9823)$ and frequency $(\mathrm{p}=0.9293)$ domains of the questionnaires. 


\section{Discussion}

In recent large Phase III clinical trials, mirabegron has demonstrated significant efficacy over placebo in treating the symptoms of $\mathrm{OAB}$, including micturition frequency, urgency incontinence, and urgency ${ }^{25-26}$. Given IC/BPS similar overlying symptomatology with OAB in terms of urgency related symptoms, we hypothesized that mirabegron may also play a role in treating that subset of symptoms in the IC/BPS population. The analysis of our prospectively collected real life clinical practice data from a single centre IC/BPS patient population shows that mirabegron may improve urgency symptoms in patients with IC/BPS however, showed no significant improvement in frequency or pain symptoms. Furthermore, no significant improvement was noted in the total scores of the disease specific ICSI, ICPI, and PUF questionnaires.

Although the symptoms of urgency and frequency are described by both OAB and IC populations, there may be a qualitative difference in the way these symptoms are experienced. IC/BPS patients may experience a more constant urge to void as opposed to the ICS definition of a "compelling need to urinate which, is difficult to postpone. ${ }^{52,53}$ The prototypical OAB patients urge to void is driven to prevent episodes of incontinence however in the IC/BPS population, patients void to stop or to relieve pain. Overall the patients perceive some improvement in urgency not corroborated by the specific urgency associated questions. As mirabegron is primarily targeted at motor relaxation of the detrusor muscle and not bladder sensation, this may explain mirabegron's efficacy at treating the urgency in the OAB population compared to the IC/BPS subgroup and therefore the limited benefit seen in this study.

Pain and discomfort is also a significant symptom in IC/BPS. However, our knowledge of the afferent mechanisms that determine perceptions of lower urinary tract symptoms is limited. It is known that afferent sensory receptors of the urothelium of the bladder communicate with the central nervous system via finely mylenated a-delta fibers and unmylenated c-fibers. Adelta fibers sense bladder filling and tension within the bladder wall whereas $C$ fibers transmit discomfort or pain in response to excessive stretching of the bladder wall. A study performed by Aizawa et al. ${ }^{27}$ found that mirabegron can inhibit bladder afferent activity of A $\delta$-fibers and cfibers in a dose dependent fashion. Via inhibition of these afferent pain fibers, it was anticipated that mirabegron may decrease the sensation of pain associated with IC/BPS. Despite this, we were not able to show any significant reduction in pain following mirabegron administration. It is conceivable that pain reduction by other IC/BPS treatments could potentiate the benefits in terms of urgency improvement, but our analysis was not powered to show such an effect.

The findings of this study must be interpreted in the context of real life clinical setting rather than a prospective clinical trial design. Given then real world design, this study was not without limitations which include the limited sample size and subsequent low power. Furthermore, as this was a real world evaluation, patients were enrolled were on concurrent medications in addition to starting mirabegron therapy as well as unblinded to treatment. The 
effect of synergistic treatment with mirabegron is difficult to ascertain given the heterogeneity in treatment baseline. Furthermore our patient population has previously been assessed and optimally treated prior to starting mirabegron as an add-on. Perhaps mirabegron may more effective in newly diagnosed IC/BPS patients with early storage symptoms and mild pain.

Given the results of our current observations, we cannot recommend a large double blind placebo-controlled clinical trial to assess the efficacy of mirabegron as a monotherapy and it would be difficult to design a study evaluating it as an add-on or adjuvant treatment. That being said, it seems reasonable to consider initiating mirabegron as an adjuvant treatment in patients who continue to have bothersome urgency despite standard IC/BPS therapy.

\section{Conclusion}

Our study was the first evaluation of daily low-dose mirabegron therapy for IC/BPS. Despite the limitations of this analysis, the outcomes suggest that mirabegron currently plays a limited role for the treatment of urgency but not the pain associated with IC/BPS. 


\section{References}

1. Parsons CL, Benson G, Childs SJ et al. A quantitatively controlled method to study prospectively interstitial cystitis and demonstrate the efficacy of pentosane polysulfate. $J$ Urol 1993;150:845.

2. Bladder Research Progress Review Group: Overcoming Bladder Disease: A Strategic Plan for Research. Bethesda, MD: National Institute of Diabetes and Digestive and Kidney Diseases of the National Institutes of Health 2002;8.

3. Hanno PM, Landis JR, Matthews-Cook Y et al. The diagnosis of interstitial cystitis revisited: lessons learned from the National Institutes of Health Interstitial Cystitis Database study. J Urol 1999;161:553.

4. Abrams P, Cardozo L, Fall M, et al. The standardisation of terminology of lower urinary tract function: report from the Standardisation Sub-committee of the International Continence Society. Am J Obstet Gynecol 2002;187:116-26.

5. Nickel JC: Words of wisdom. Re: clinical phenotyping in chronic prostatitis/chronic pelvic pain syndrome and interstitial cystitis: a management strategy for urologic chronic pelvic pain syndromes. EurUrol 2009;56:881.

6. Shoskes DA, Nickel JC, Rackley RR et al. Clinical phenotyping in chronic prostatitis/chronic pelvic pain syndrome and interstitial cystitis: a management strategy for urologic chronic pelvic pain syndromes. Prostate Cancer Prostatic Dis 2009;12:177.

7. Nickel JC, Tripp DA. International Interstitial Cystitis Study Group. Clinical and Psychological Parameters Associated with Pain Pattern Phenotypes in Women with Interstitial Cystitis/Bladder Pain Syndrome J Urol 2015;193:138.

8. Nickel JC, Tripp DA, Pontari M et al. Interstitial cystitis/painful bladder syndrome and associated medical conditions with an emphasis on irritable bowel syndrome, fibromyalgia and chronic fatigue syndrome. J Urol 2010;184:1358.

9. Peters KM, Girdler B, Carrico DJ et al. Painful bladder syndrome/interstitial cystitis and vulvodynia: a clinical correlation. Int Urogynecol 2008;19:665.

10. Rodriguez MA, Afari N, Buchwald DS. Evidence for overlap between urological and nonurological unexplained clinical conditions. J Urol 2009;182:2123.

11. Hanno PM, Burks DA, Clemens JQ et al. AUA guideline for the diagnosis and treatment of interstitial cystitis/bladder pain syndrome. J Urol, 2011;185:2162

12. Dell JR, Parsons CL. Multimodal therapy for interstitial cystitis. J Reprod Med 2004;49:243-52.

13. Tincello DG, Walker AC. Interstitial cystitis in the UK: results of a questionnaire survey of members of the Interstitial Cystitis Support Group. Eur J Obstet Gynecol Reprod Biol 2005;118:91. 
14. Diggs C, Meyer WA, Langenberg P et al. Assessing urgency in interstitial cystitis/ painful bladder syndrome. Urology 2007;69:210.

15. Greenberg P, Tracy JK, Meyer WA et al. Short interval between symptom onset and medical care as an indication of rapid onset of interstitial cystitis/painful bladder syndrome. BJU Int 2007;100:599.

16. Chapple CR, Khullar V, Gabriel Z et al. The effects of antimuscarinic treatments in overactive bladder: An update of a systematic review and meta-analysis. Eur Urol 2008;54:543-62.

17. Minaglia S, Özel B, Bizhang R et al. Increased prevalence of interstitial cystitis in women with detrusor overactivity refractory to anticholinergic therapy. Urology 2005;66:702-6.

18. Chung MK, Butrick C, Chung RP et al. The overlap of interstitial cystitis and overactive bladder. J Pelvic Med Surg. 2003;9:244.

19. Andersson KE, Arner A. Urinary bladder contraction and relaxation: Physiology and pathophysiology. Physiol Rev 2004;84:935.

20. Michel MC, Vrydag W. $\alpha 1$-, $\alpha 2$ - and $\beta$-adrenoceptors in the urinary bladder, urethra and prostate. Br J Pharmacol, 2006;147:88.

21. Otsuka A, Shinbo H, Matsumoto R et al. Expression and functional role of $\beta$ adrenoceptors in the human urinary bladder urothelium. Naunyn Schmiedebergs Arch Pharmacol, 2008;377:473.

22. Wuest M. Catecholamines relax detrusor through $\beta 2$-adrenoceptors in mouse and $\beta 3$ adrenoceptors in man. J Pharmacol Exp Ther, 2009;328:213.

23. O’Leary M, Sant G, Fowler F et al. The interstitial cystitis symptom index and problem index. Urology 1991;49:58-63.

24. Brewer M, White W, Klein F et al. Validity of Pelvic Pain, Urgency, and Frequency questionnaire in patients with interstitial cystitis/painful bladder syndrome. Urology 2007;70:646-9.

25. Herschorn S, Barkin J, Castro-Diaz D et al. A Phase III, randomized, double blind, parallel-group, placebo-controlled, multicentre study to assess the efficacy and safety of the b3 adrenoceptor agonist, mirabegron in patients with symptoms of overactive bladder. Urology 2013;81:12.

26. Nitti V, Khullar V, van Kerrebroeck P et al. Mirabegron for the treatment of overactive bladder: A prespecified pooled efficacy analysis and pooled safety analysis of three randomised, double-blind, placebo-controlled, phase III studies. Int J Clin Pract 2013;67:619-32. 
27. Aizawa N, Homma Y, Igawa Y. Effects of mirabegron, a novel $\beta 3$-adrenoceptor agonist, on primary bladder afferent activity and bladder microcontractions in rats compared with the effects of oxybutynin. Eur Urol. 2012;2:1165-73.

28. Lai HH, Vetter J, Jain $\mathrm{S}$ et al. The overlap and distinction of self-reported symptoms between interstitial cystitis/bladder pain syndrome and overactive bladder: a questionnairebased analysis. The Journal of Urology, 2014;192:1679-85. 
Figures and Tables

Table 1. Patient demographics $(n=23)$

\begin{tabular}{|l|c|c|c|c|}
\hline & Mean & SD & Median & IQR \\
\hline Age & 48.96 & \pm 13.5 & 49 & 20.5 \\
\hline Symptom duration (months) & 10.47 & \pm 10.8 & 5 & 13.5 \\
\hline Months between visits & 6.9 & \pm 4.0 & 6 & 3.5 \\
\hline Sex & & & & \\
\hline$\quad$ Female & 22 & $95.6 \%$ & & \\
\hline$\quad$ Male & 1 & $4.4 \%$ & & \\
\hline Race & & & & \\
\hline Caucasian & 22 & $95.6 \%$ & & \\
\hline Other & 1 & $4.4 \%$ & & \\
\hline
\end{tabular}

IQR: interquartile range; SD: standard deviation.

\begin{tabular}{|c|c|c|}
\hline Therapy & $\mathbf{n}$ & $\%$ \\
\hline Diet modifications & 19 & 82.6 \\
\hline Antidepressants & 8 & 34.8 \\
\hline Pentosan polysulfate & 8 & 34.8 \\
\hline Pelvic floor physiotherapy & 4 & 17.4 \\
\hline Massage/yoga & 3 & 13.0 \\
\hline Psychotherapy pain program & 2 & 8.7 \\
\hline Gabapentinoids & 2 & 8.7 \\
\hline Pyridium & 2 & 8.7 \\
\hline Lidocaine $^{1}$ & 1 & 4.3 \\
\hline Sodium citrate & 1 & 4.3 \\
\hline Botox injections ${ }^{2}$ & 1 & 4.3 \\
\hline
\end{tabular}

${ }^{1}$ Started after mirabegron administration. ${ }^{2}$ Stopped prior to completion of study. 
Table 3. Total scores before and after mirabegron treatment $(n=23)$

\begin{tabular}{|l|l|c|c|c|c|c|}
\hline & & \multicolumn{2}{|c|}{ Before treatment } & \multicolumn{2}{c|}{ After treatment } & p \\
\hline \multirow{4}{*}{ Total scores } & & Median & IQR & Median & IQR & \\
\cline { 2 - 8 } & ICSI total & 12 & 6 & 12 & 5.5 & 0.554 \\
\cline { 2 - 8 } & ICPI total & 10 & 3.5 & 10 & 5 & 0.313 \\
\cline { 2 - 8 } & PUF total & 18 & 6 & 19 & 8.5 & 0.991 \\
\cline { 2 - 8 } & Symptom score total PUF & 13 & 4.5 & 12 & 5.5 & 0.834 \\
\cline { 2 - 8 } & Bother score total PUF & 6 & 3 & 6 & 3 & 0.894 \\
\hline
\end{tabular}

ICPI: Interstitial Cystitis Problem Index; ICSI: Interstitial Cystitis Symptom Index; IQR: interquartile range; PUF: Pelvic Pain and Urgency/Frequency Patient Symptom Scale.

Table 4. Symptom-specific scores before and after mirabegron treatment $(n=23)$

\begin{tabular}{|c|c|c|c|c|c|c|}
\hline & & $\begin{array}{l}\text { Befo } \\
\text { treatm }\end{array}$ & & $\begin{array}{r}\text { Aft } \\
\text { treatn }\end{array}$ & & $\mathbf{p}$ \\
\hline & & Median & IQR & Median & IQR & \\
\hline Pain & Pain NRS & 6 & 3.5 & 6 & 4 & 0.9823 \\
\hline Frequency & Frequency NRS & 7 & 3.5 & 7 & 3.5 & 0.9293 \\
\hline & $\begin{array}{l}\text { Urinate less than } 2 \text { hours after coid } \\
\text { ICSI }\end{array}$ & 4 & 2 & 4 & 3 & 0.8615 \\
\hline & Frequent urination day ICPI & 3 & 0.5 & 3 & 2 & 0.7897 \\
\hline & Bathroom count per day PUF & 2 & 2 & 2 & 1.5 & 0.5336 \\
\hline Urgency & Urgency NRS & 7 & 2 & 5 & 3 & 0.0717 \\
\hline & Urinate with no warning ICSI & 4 & 1.5 & 2 & 3 & 0.0102 \\
\hline & Need urinate little warning ICPI & 3 & 1 & 2 & 2 & 0.6209 \\
\hline & Urgency rating PUF & 2 & 1.5 & 1 & 2 & 0.6541 \\
\hline
\end{tabular}

ICPI: Interstitial Cystitis Problem Index; ICSI: Interstitial Cystitis Symptom Index; IQR: interquartile range; PUF: Pelvic Pain and Urgency/Frequency Patient Symptom Scale. 